\title{
The Applications of Current Comparators in the Measurements on High Voltage Insulation
}

\author{
Yi-jun FEI ${ }^{1}, X u D_{D N G^{2}}$, Jie CHEN ${ }^{1}$, Huan ZHENG ${ }^{2}$, Jing-ying CAO ${ }^{1}$, Zhong-hua $\mathrm{LI}^{2}$ \\ 1 Jiangsu Electric Power Research Institute, Nanjing Jiangsu \\ ${ }^{2}$ College of Electrical \& Electronic Engineering, Harbin University of Science and Technology
}

\begin{abstract}
This paper describes the basic structure of the current comparator used for high voltage insulation measurements. Further applications for the current comparator in high voltage insulation are investigated and developed. A measuring system for the measurement of harmonics in the loss current of water tree aged insulation is described, as well as the principles to measure partial discharges with the current comparator bridge. A new system for the measurement of the DC component in the leakage current of insulation is developed and presented. The results of experiments on XLPE cable insulation are also given.
\end{abstract}

\section{Introduction}

Current comparators have been widely used for measurement and calibration purposes in high voltage engineering with the main function being the measurement of the dielectric loss factor $\tan \delta$ of high voltage insulation. In the early 1990's, Japanese and Canadian researchers developed the current comparator bridge further to apply it in laboratory tests for the measurement of harmonics in theloss current of water tree aged XLPE cable insulation[1,2].

In this study, a current comparator based high voltage capacitance bridge was primarily designed and built to measure the harmonics produced by water trees in XLPE cable insulation. During the experiments to measure the harmonics in a service-aged cable, it was found that the current comparator based high voltage bridge could be used as an input unit for measuring partial discharges occurring in the insulation.

A new and important application developed in this study is to use the current comparator as the basis of a measuring system to measure the DC component in the 1eakage current of insulation with the application of an $\mathrm{AC}$ voltage. In the system the AC component, that is much larger than the DC component to be measured, is filtered out and the detection winding of the current comparator is only used to monitor the conditions for the AC component to be filtered or balanced out[3].

\section{Basic structure of current comparator used in high voltage}

The basic structure of the current comparator core used for high voltage insulation measurements is shown in Figure 1.

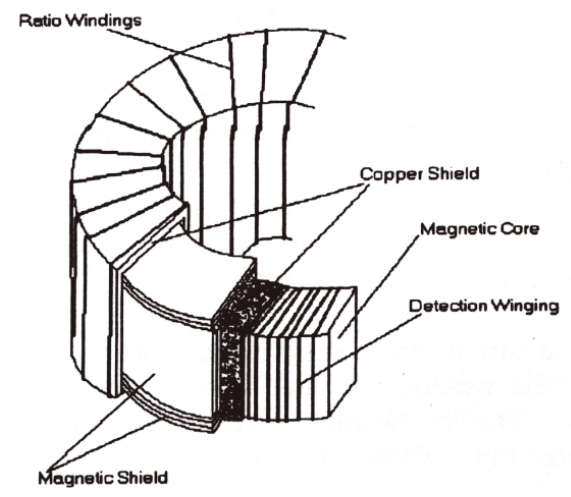

Figure 1. Basic structure of current comparator core

For applications in high voltage, the current comparator should be carefully designed to obtain high sensitivity and special care must be taken to get good interference suppression as well, as the signals to be measured during high voltage experiments on insulation are small and interference levels high[4]. The magnetic core must have a high permeability and the detection winding must be wound with a high density onto the core to ensure maximum sensitivity. The sensitivity of a current comparator is proportional to the permeability of the core and the density of the windings[5]. The detection winding has 1500 turns and is connected to a null detector and other measuring instruments depending on the application. To eliminate interference, two copper shields and a magnetic shield are used to screen the detection winding. 


\section{Measurement of harmonics}

In the early of 1990 's, it was reported researchers that water trees in XLPE cable insulation can produce harmonics in the loss current when $\mathrm{AC}$ high voltage is applied to the cable[1,2].

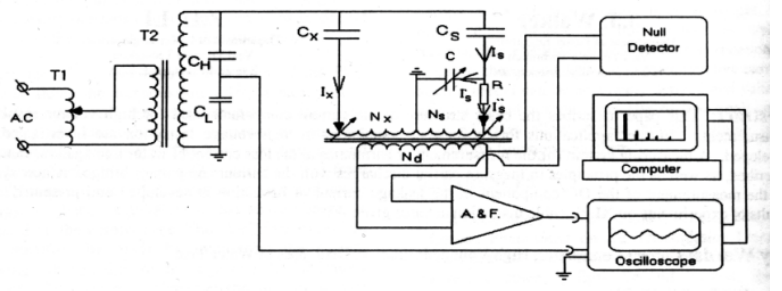

Figure 2. System to measure harmonic distortion in loss current

The basic configuration of the measuring system developed in this study is shown in Figure 2, This system consists of a current comparator based high voltage capacitance bridge and a computer measuring and analysis system.

In the current comparator based, high voltage bridge, the ratio windings $\mathrm{Nx}$ and Ns were wound with 1000 turns[6]. The Ns winding, which is connected to a standard high-voltage low-loss gas-insulated capacitor Cs, is wound so that single-turn increments are available. The $\mathrm{Nx}$ winding, which is connected to the capacitance $\mathrm{Cx}$ to be measured, have taps at $1,2,5,10,20, \ldots, 500$, and 1000 turns. The working principle of the bridge can be seen form the vector diagram shown in Figure 3.

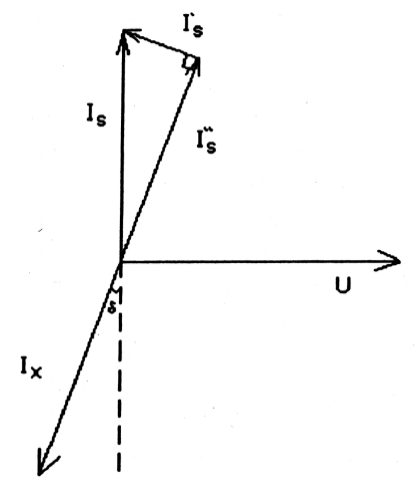

Figure 3. Vector diagram for bridge when balanced

From Figure 3, drawn for balanced conditions, the following can be derived:

$$
\begin{aligned}
& \tan \delta=\omega R C \\
& C_{X}=C_{s} \frac{N_{s}}{N_{x}}
\end{aligned}
$$

In which, $\tan \delta$ is the dielectric loss factor of sample to be measured, and $\omega$ is the angular frequency.

From equations (1) and (2), it can be seen that the current comparator bridge can be used to measure $\tan \delta$ and the capacitance of the insulation, which is the traditional application for the current comparator bridge.

The amplifier, specifically developed for these measurements, has a gain of 10000 , and the filter is a low pass type with a cut-off frequency of $1000 \mathrm{~Hz}$. The computer is equipped with a 16 -bit $\mathrm{A} / \mathrm{D}$ measuring card with sampling frequency of $5000 \mathrm{~Hz}$. The data is stored in the computer and analysed with a signal processing software program.

The experimental results of measurements on a $5 \mathrm{kV}$, service-aged, XLPE cable aged with water trees are given in Figure 4. The cable was soaked in water and tested, then allowed to dry out and tested again. These results clearly shows that the harmonics in the loss current produced by water trees in the insulation, increased when the cable were soaked and decreased when the cable were allowed to dry out.
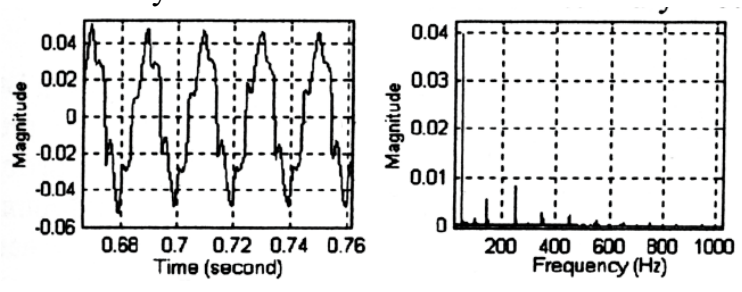

(a) The loss current and its FFT for service aged cable with water at $9 \mathrm{kV}$

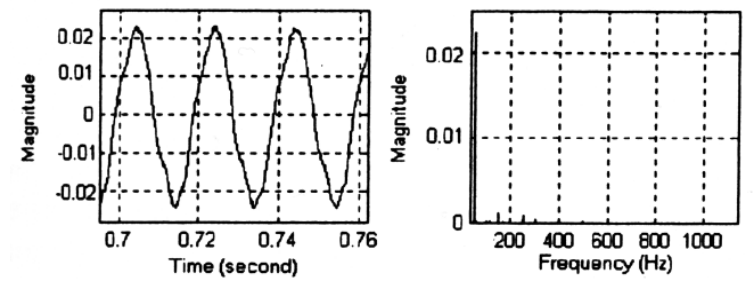

(b) The loss current and its FFT for service aged cable without water at $9 \mathrm{kV}$

Figure 4. Harmonics in loss current of service aged cable with and without water

\section{Partial discharge measurement}

While the measurements of the harmonics in the loss current were in progress, it was found that the current comparator can be used as an input unit for partial discharge (PD) measurement. In the application on PD measurement, the detection winding must be connected directly to an oscilloscope or partial discharge detector[7]. The reason why the detection winding must be connected directly to the oscilloscope is that the filter used for harmonic measurement is a low-pass filter with a cut-off frequency of $1000 \mathrm{~Hz}$. For the PD signals, the current comparator bridge circuit is equivalent to the $\mathrm{PD}$ measuring circuit shown in Figure 5, that is, the bridge is equivalent to the RLC input unit. 


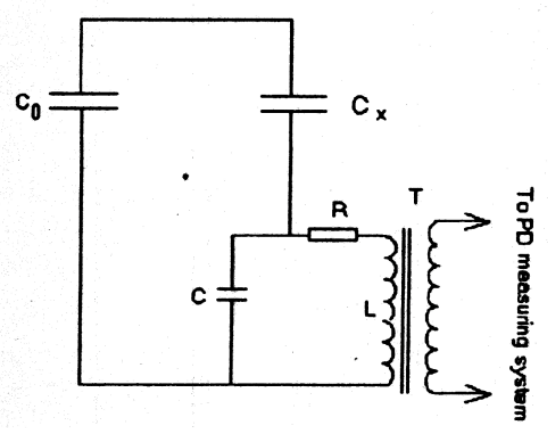

Figure 5. Equivalent circuit for PD measurement

To record the experimental results of the PD measurements on a service-aged cable, the detection winding was connected to the computer measuring system. The partial discharges in the service-aged XLPE cable were measured at $8 \mathrm{kVp}$. The partial discharge signals and the applied voltage on the cable are shown in Figure 6. The PD signals in the cable were determined to be $60 \mathrm{pC}$, as compared to a portable calibrator.
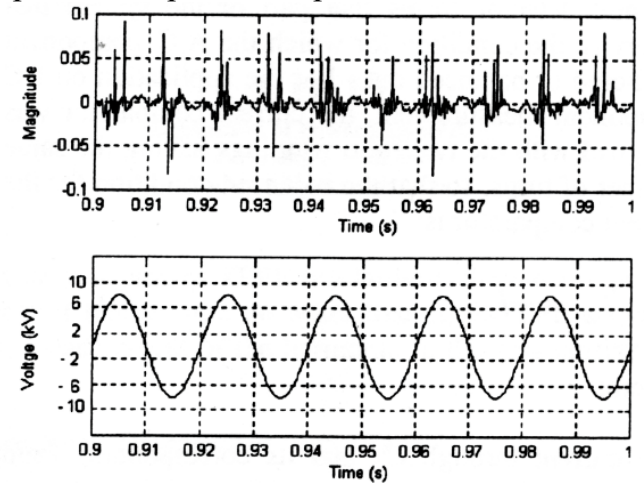

Figure 6. PD signals and applied voltage

It must be mentioned that some signals appearing on the oscilloscope are not shown in Figure 6 as the computer measuring system with a sampling frequency of $5000 \mathrm{~Hz}$ is not suited for PD measurement. This result is significant as the possibility now exist that PD and $\tan \delta$ of insulation can be measured simultaneously by one measuring system.

\section{DC component measurement}

It has been reported that the DC component of the current in XLPE insulation while an AC voltage is applied to the insulation can be the result of water trees and water in the insulation material[3,6]. The measurement of the DC component of the current in the insulation can therefore be developed as a method for the diagnosis of water tree in XLPE-insulated cables. The D.C. component measuring system that was developed in this study is shown in Figure 7.

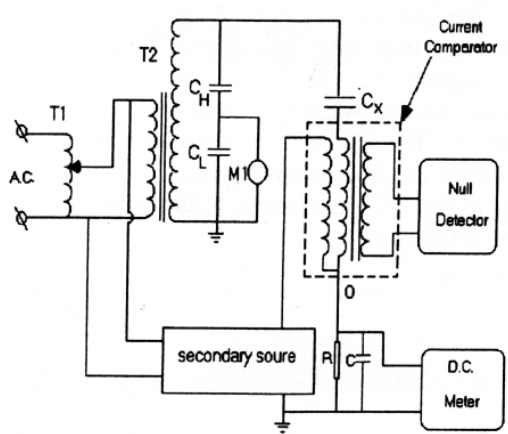

Figure 7. System to measure the D.C component of the leakage current

As the main component in this measuring system, a current comparator is used and connected to a high sensitivity null detector. The current comparator and the nul1 detector forms that part of the system that supervise the condition for which the A.C. component is filtered or balanced out. For the application on DC component measurement, the current comparator was designed with the two ratio windings having the same number of turns, so that the balanced condition for the current comparator is:

$$
I_{X}=-I_{S}
$$

Where:

$\mathrm{Ix}=$ current through $\mathrm{Cx}$ and its corresponding ratio winding to the common point ' $\mathrm{O}$ '; and $\mathrm{Is}=$ current from secondary source to the common point ' $\mathrm{O}$ '.

The number of turns of the ratio windings depends on the capacitance of the sample to be measured and the required sensitivity for the specific measurement. For laboratory applications where measurements are done on short lengths of cable, the number of turns for the ratio windings can be chosen between50 and 200. For field tests where the capacitance of the sample is much larger, the ratio windings can be designed to have approximately 5turns.

The D.C. component produced by water trees in XLPE cable insulation can now be measured across the sampling resistance $R$. The value of $R$ is $1 M \Omega$,the sensitivity of the D.C. meter is $1 \mathrm{mV}$, giving this system a sensitivity of $1 \mathrm{nA}$ for D.C.current measurements.

To verify that the measurement of the D.C. component in the leakage current can be developed as an effective diagnostic method for evaluating water trees[8], comparative measurements were done on the service aged cable and a new cable. Tests were done on the cables after they were submerged in water to allow water ingress between the core screen and the insulation and between the conductor screen and the insulation. The cables were then allowed to dry out after which the tests were repeated. The comparative results of the DC component at different voltages are shown in Figure 8. 


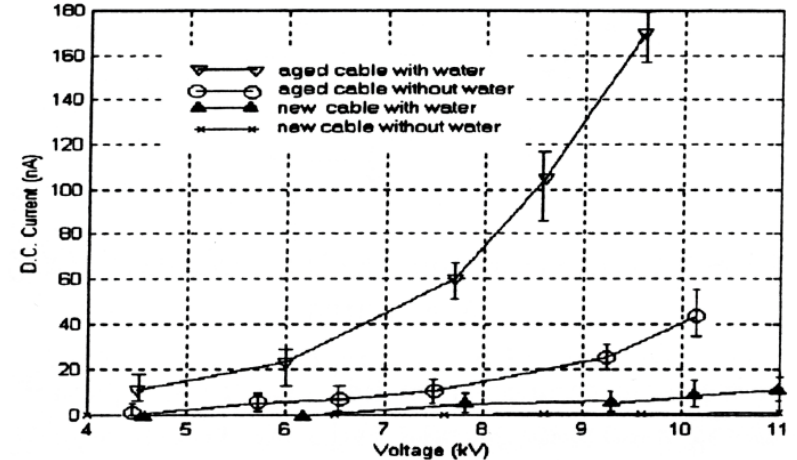

Figure 8. D.C. components of cable at different voltages

The experimental results in Figure 8 shows that the DC component is higher in the service-aged cable presoaked in water than in the same cable after a period when drying out of water was allowed. It is also found that the D.C. component can be measured in the new cable that was soaked in water with no DC component in the dry new cable.

\section{Conclusion}

In this study, new application of current comparator in the measurement of high-voltage insulation were studied and developed. This study has not been completed and further results will be presented in the future.

\section{Acknowledgement}

The authors would like to thank the State Grid Electric Power Research Institute in Jiangsu Province for their financial support.

\section{References}

1. Ziabakhsh S, Alavi-Rad H, Alavi-Rad M, et al. The design of a low-power high-speed current comparator in $0.35-\mu \mathrm{m}$ CMOS technology[C] Quality of Electronic Design. ISQED 2009. Quality Electronic Design IEEE(2009)

2. Takahashi K, Yagi K. Development of AC current comparator based on coaxial cable for enlarging measurement frequency range up to $10 \mathrm{kHz}[\mathrm{C}]$ Precision Electromagnetic Measurements Digest. CPEM 2008. Conference(2008)

3. Zhang L. A High-speed Comparator for a 12-bit 100MS/s Pipelined ADC[C] Intelligent Computation Technology and Automation (ICICTA), 2011 International Conference on IEEE(2011)

4. Illias, H.; Teo Soon Yuan; Bakar, A.H.A.; Mokhlis, H. Chen, G.; Lewin, P.L., "Partial discharge patterns in high voltage insulation," in Power and Energy (PECon), 2012 IEEE International Conference(2012)

5. Moore, W.J.M and Miljanic, P.N,The Current Comparator. Peter PeregrinusLtd., IEEE Electrical Measurement Series 4(1988)

6. Zheng $\mathrm{X}$. Q and Tu DM. Frequency different respond of ULFSW-MVW superposition voltage in water treeing degraded XLPE cable insulation. High Voltage Engineering(2000)

7. Dodd, S.J.; Chalashkanov, N.M.; Fothergill, J.C. "Partial discharge patterns in conducting and nonconducting electrical trees," in Solid Dielectrics (ICSD), 2010 10th IEEE International Conference (2010)

8. C.L.Wadhwa, High Voltage Engineering, New Age international(p) Limited, Publishers(1997) 